\title{
Active deformation of apoptotic intestinal epithelial cells with adhesion-restricted polarity contributes to apoptotic clearance
}

\author{
Fengchao Wang ${ }^{1}$, Fengjun Wang ${ }^{2}$, Zhongmin Zou ${ }^{3}$, Dengqun $\mathrm{Liu}^{1}$, Junping Wang ${ }^{1}$ and Yongping Su ${ }^{1}$
}

Dying epithelial cells are thought to be squeezed out of the epithelium by the contraction of an actomyosin ring formed in live neighboring cells, which simultaneously closes any potential gap, thereby maintaining the integrity of the epithelial layer. The shrinkage and contraction of apoptotic cells contribute little to the extrusion process. In contrast, the clearance of dying intestinal columnar epithelial cells in vivo usually leaves a transient gap via an unknown mechanism. By using freshly isolated small intestinal villus units with or without basal lamina, we found that the nucleus of apoptotic enterocytes moved apically until they budded off, leaving the cytoplasmic residue in the transient gap. Apical polarity of nucleus movement was restricted unless the basal lamina was artificially removed. F-actin mainly accumulated in apoptotic cells rather than neighboring live cells, even after the addition of resistance force against extrusion. The actin accumulation in apoptotic cells does not depend on the living state of neighboring cells. Apoptotic cells can complete the shedding process when neighboring a goblet cell, as the majority of space is occupied by mucin granules and the cytoplasm consists of intermediate filaments and microtubules, but lacks F-actin. We found that the elongation and deformation of apoptotic cells depend on the stretching force generated inside the cell, rather than the force generated by neighboring cells extending. Our findings clearly demonstrate that intestinal epithelial shedding does not depend on the formation and contraction of an actomyosin ring in live neighboring cells. Apoptotic epithelial cells may undergo an active process of cell deformation with adhesion-restricted polarity, which may contribute to maintaining barrier function during a high rate of cellular turnover.

Laboratory Investigation (2011) 91, 462-471; doi:10.1038/labinvest.2010.182; published online 1 November 2010

KEYWORDS: apoptosis; clearance; deformation; epithelial cell; polarity

The clearance of apoptotic epithelial cells is critical to shaping an embryo and maintaining homeostasis in adult tissues. Besides the phagocytic clearance of apoptotic cells, 'apoptotic cell extrusion' is thought to be another process for clearance of apoptotic epithelial cells. ${ }^{1,2}$ Apoptotic volume decrease (AVD) or cell shrinkage is thought to be a ubiquitous characteristic of programmed cell death and occurs in nearly all reported examples of apoptosis. ${ }^{3-6}$ However, this shrinkage and contraction of apoptotic epithelial cells contribute very little to their extrusion from the epithelial sheet. Extrusion depends on the circumferential contraction of an actomyosin ring formed in live neighboring cells, which simultaneously closes any gap resulting from a dying cell's exit. $^{7}$
The extrusion of apoptotic cells was mostly found to be processed apically in the epithelia encasing vertebrate embryos and adult organs. ${ }^{2,7}$ Basal extrusion was observed occasionally in vertebrates and more commonly in Drosophila embryonic epithelia. ${ }^{2,8,9}$ Recently, nearly $20 \%$ of basal extrusion was observed in ultraviolet-irradiated MadinDarby canine kidney (MDCK) cells. Basal and apical extrusion polarity is determined by the localization of actomyosin contraction in the apices or basolateral surfaces. To extrude the apoptotic cell apically, the microtubules in neighboring cells need to be reoriented, and target p115 RhoGEF to the basolateral site and activate actin/myosin. ${ }^{2}$

Rapid and frequent elimination of the effete and damaged epithelial cells is essential to maintain small intestinal barrier

\footnotetext{
${ }^{1}$ Institute of Combined Injury, State Key Laboratory of Trauma, Burns and Combined Injury, College of Preventive Medicine, Third Military Medical University, Chongqing, China; ${ }^{2}$ Institute of Burn Research, State Key Laboratory of Trauma, Burns, and Combined Injury, Southwest Hospital, Third Military Medical University, Chongqing, China and ${ }^{3}$ Department of Chemical Defenses and Toxicology, College of Preventive Medicine, Third Military Medical University, Chongqing, China Correspondence: Dr Y Su, MD, PhD, or Professor J Wang, MD, PhD, Institute of Combined Injury, Third Military Medical University, 30 Gaotanyan Road, Shapingba District, Chongqing, 400038, China. E-mail: yongping.su@yahoo.com.cn or wangjunp@yahoo.com

Received 6 April 2010; revised 12 September 2010; accepted 24 September 2010
} 
function. ${ }^{10-12}$ In vivo observations have revealed that most of the resulting vacant spaces could be transiently maintained during and after cell extrusion. ${ }^{13,14}$ Based on existing theories, it remains unclear how the neighboring cells squeeze the apoptotic cell out and at the same time a gap remains. The determinant of extrusion polarity in intestinal epithelial cells also remains elusive. Here, we recapitulate extrusion that leaves these gaps by culturing mouse intestinal epithelium with and without basal lamina and explore the mechanisms of this unique apoptotic cell clearance.

\section{MATERIALS AND METHODS Mice}

Male C57BL/6 mice (18-22 g) were obtained from the Shanghai Laboratory Animal Center (Chinese Academy of Sciences). All animal experiments were performed with the approval of the animal ethics committee of the Third Military Medical University (China).

\section{Isolation of Intestinal Mucosa and Villus Units without Basal Lamina}

The duodenum and jejunum were removed from each animal and flushed with cold d-Hank's solution $\left(4{ }^{\circ} \mathrm{C}\right)$ containing $0.5 \mathrm{mM}$ dithiothreitol to remove the luminal contents. Intestinal tracts were then everted and the intestinal mucosa was scraped with a sharp blade. The collected mucosa was suspended in $30 \mathrm{ml}$ cold d-Hank's solution, and collected by sedimentation for $20 \mathrm{~s}$. Sedimentation was repeated three times and the mucosal enrichment was suspended in ice-cold Dulbecco's modified Eagle's medium (DMEM; Invitrogen, Carlsbad, CA, USA). The villus units without basal lamina were isolated and enriched as previously described. ${ }^{15}$

\section{Tissue Culture}

After being washed with ice-cold Hank's solution, the villus units with or without basal lamina were gently resuspended in ice-cold DMEM containing 50 units $/ \mathrm{ml}$ penicillin, $0.5 \mathrm{mg} / \mathrm{ml}$ gentamycin and $5 \%$ fetal bovine serum (Hao Yang Biological Manufacture, Tianjin, China), and incubated for 7-15 min in six-well culture plates $(100 \mu \mathrm{l} /$ well for villus without basal lamina, and $200 \mu \mathrm{l} /$ well for villus with lamina propria) at $37^{\circ} \mathrm{C} / 5 \% \mathrm{CO}_{2}$. In the system for adding resistance force against cell extrusion, the ice-cold villus-crypt units were embedded matrigel (BD Biosciences, San Jose, CA, USA) or cultured in warm media in the same 24-well plates. The plate was incubated for $2 \mathrm{~min}$ in a $37^{\circ} \mathrm{C}$ water bath and was then maintained for $5-8 \mathrm{~min}$ at $37^{\circ} \mathrm{C} / 5 \% \mathrm{CO}_{2}$.

\section{Time-Lapse Imaging and Confocal Microscopy}

The villus units scraped from the duodenum and jejunum of BL6 mice were cultured on a Leica AF6000 Live Cell Imaging Workstation (Wetzlar, Germany) at $37{ }^{\circ} \mathrm{C} / 5 \% \mathrm{CO}_{2}$. Phase-contrast images were taken at intervals of $10 \mathrm{~s}$. When recording the time-lapse photography, the time spent to locate the target villus unit and to operate the software before images were acquired was taken into account. After a 7-min incubation, the villus units were sampled and fixed in Histochoice ${ }^{\mathrm{TM}} \mathrm{MB}$ (Amereson, Solon, OH, USA) overnight at $4{ }^{\circ} \mathrm{C}$. The villus units were then washed twice with $70 \%$ ethanol and three times with phosphate-buffered saline (PBS). After nuclear staining with DAPI $(100 \mathrm{ng} / \mathrm{ml}$ in PBS), the villus units were immersed in PBS and observed with a confocal microscope (Zeiss LSM510 META, Oberkochen, Germany) with a planapochromatic $\times 400.75$ NA objective. Consecutive confocal images were generated at different Z-stack intervals. The micrographs were processed with Adobe Photoshop CS2 8.0 and assembled with Adobe Illustrator CS2 12.0.

\section{Electron Microscopy}

Sampled villus were fixed with $2.5 \%$ glutaraldehyde at $4{ }^{\circ} \mathrm{C}$ overnight, post-fixed with $1 \% \mathrm{OsO}_{4}$ for $30 \mathrm{~min}$ and then processed as previously described. ${ }^{16}$

\section{Immunohistochemistry}

For immunohistochemistry, the villus units were sedimented for $30 \mathrm{~s}$ on ice. The collected units were fixed with Histochoice for $12 \mathrm{~h}$ at $4{ }^{\circ} \mathrm{C}$ and were then processed as previously described. ${ }^{16}$ The antibody used for immunostaining was a rabbit anti-active caspase-3 (1:100; Cell Signaling Technology, Beverly, MA, USA).

\section{Staining Procedures for Confocal Laser Scanning Microscopy Studies}

The intestinal mucosa or the isolated villus units before and after culturing were collected by sedimentation. After removing the culture media, samples were fixed with $10 \%$ formalin for $10 \mathrm{~min}$ at $37^{\circ} \mathrm{C}$ and then for $1 \mathrm{~h}$ at $4{ }^{\circ} \mathrm{C}$. Single villus units with basal lamina were removed from fixed intestinal mucosa by micro tweezers under a stereo microscope (Zeiss STEMI SV8) and collected by sedimentation in PBS. The collected villus units with or without basal lamina were washed three times with PBS and permeabilized with $0.5 \%$ Triton X-100 in PBS for $50 \mathrm{~min}$ at room temperature. To block nonspecific binding sites, villus units were washed in blocking solution (PBS containing 10\% (v/v) goat serum and $0.1 \%$ Triton X-100) for $60 \mathrm{~min}$. The villus units were then incubated with primary antibody diluted in blocking solution for $1 \mathrm{~h}$, washed three times with PBS containing $0.1 \%$ Tween20 and then incubated with secondary antibodies. The primary antibodies used for immunostaining were mouse $\beta$-catenin antibody (1:100; Santa Cruz Biotechnology, Santa Cruz, CA, USA) and rabbit anti-active caspase-3 (1:100; Cell Signaling Technology). Actin was detected using ActinTracker Green (1:100; Beyotime, Shanghai, China). Alexa Fluor 633 goat anti-mouse (Invitrogen) and Cy3-labeled goat anti-rabbit IgG (Beyotime) were used as secondary antibodies. DNA was detected with $3 \mu \mathrm{g} / \mathrm{ml}$ DAPI. The stained 

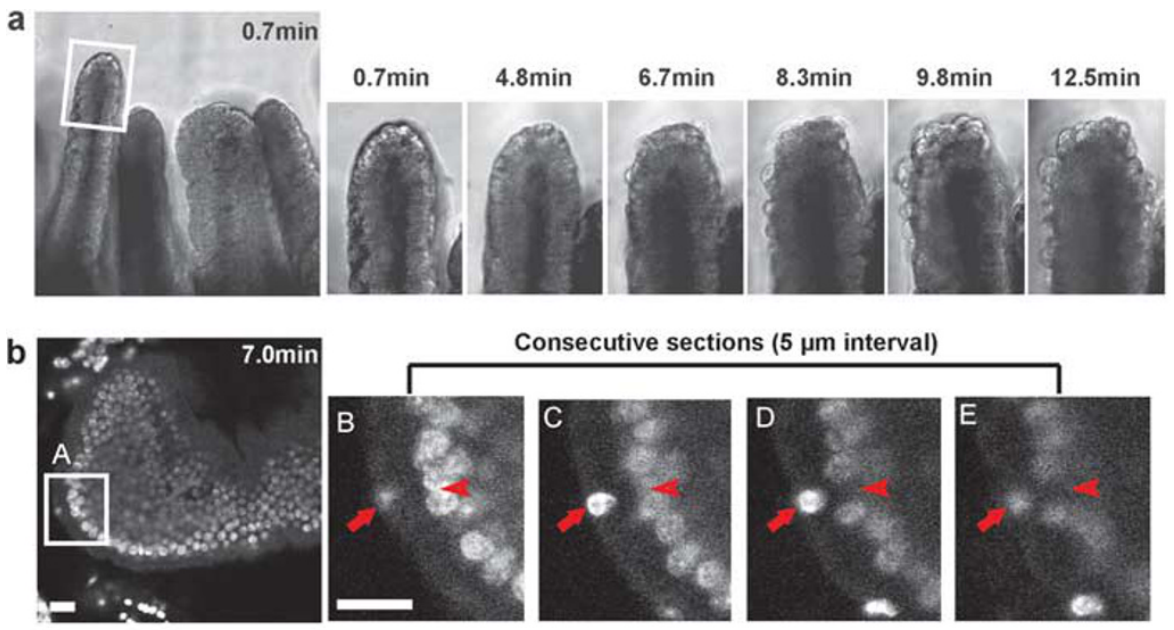

Consecutive sections (5 $\mu \mathrm{m}$ interval)

C

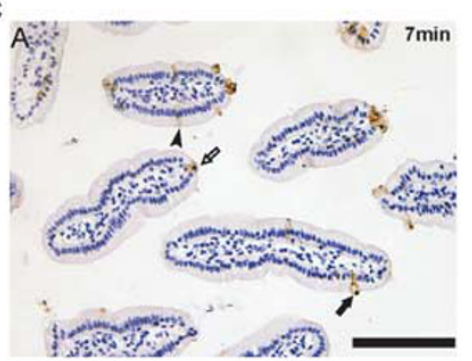

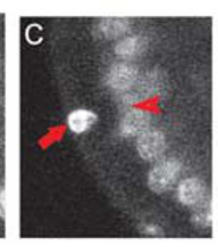
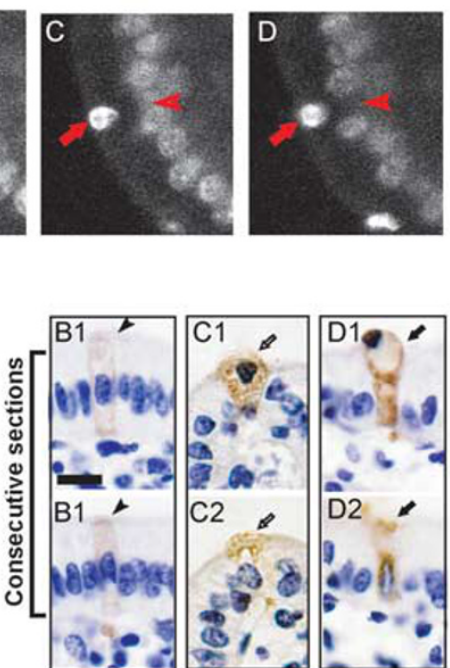

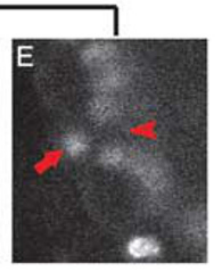

d

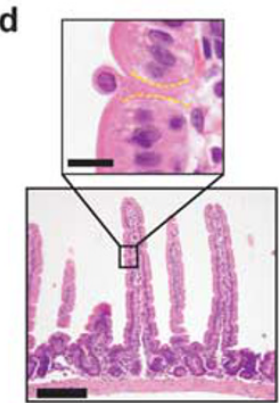

Figure 1 Cell extrusion in villus units with basal lamina. (a) Time-lapse images of epithelial extrusion in the upper villus of mouse jejunum.

(b) Representative cell extrusion in the middle phase. Left-one section of the villus tip. Right-magnified, boxed area from the left graph, following three consecutive sections with a Z-stack interval of $5 \mu \mathrm{m}$. The arrows indicate the nuclei of shedding cells; arrowheads indicate the regions devoid of nuclei, occurring beneath the shedding cells. Scale bars $=20 \mu \mathrm{m}$. (c) Representative cell shedding morphology in scraped villus units after incubation for 7 min. Shedding cells in the very early phase (arrowhead), middle phase (white arrow) and late phase (black arrow) are shown, plus the neighboring cells in consecutive sections ( $5 \mu \mathrm{m}$ intervals). (d) An example of cell shedding from normal jejunum. The boxed area shown is magnified, and the boundary of the shedding cell is marked by the dashed line. Scale bars: (c, d) $=100 \mu \mathrm{m}$; magnified image $=10 \mu \mathrm{m}$.

villus units were mounted with Antifade Mounting Medium (Beyotime). Confocal sections were taken using a Zeiss LSM 510 Meta confocal laser scanning microscope using a $\times 63$ oil lens at a resolution of $1024 \times 1024$. All images were processed further using Adobe Photoshop and Illustrator.

\section{RESULTS}

\section{Apoptotic Extrusion of Epithelial Cells with Adhesion Leaves Transient Gaps}

Both the apoptotic cell extrusion and the restitution of single-cell defects in the mouse intestinal tissues differed from that observed in cultured cells. ${ }^{13,14,17}$ To recapitulate the in vivo physiological process, the crypt-villus units with lamina propria isolated from the mouse small intestine were incubated in vitro. After incubation in vitro for 6-8 $\mathrm{min}$, the shedding cells began to bud off from the villus tip (Figure 1a) as single cells or cell sheets (Supplementary Figure S1). After a 7-min incubation, the sample was fixed and observed under a confocal microscope. The manner of shedding with gap formation (Figure 1b) was morphologically the same as previous in vivo observations. ${ }^{13}$ This transient vacancy could not be seen if the observation planes did not go through the whole body of shedding cells, and most possibly the presence of a gap-free extrusion may be improperly concluded (Figure 1b, B and C).

To further investigate the manner of extrusion and its association with caspase activation, the samples that were cultured for $7 \mathrm{~min}$ were incubated with an antibody against cleaved caspase-3. It was observed that effective cleavage of caspase- 3 was triggered before any morphological changes occurred (Figure 1c, B), and then the nucleus moved toward the apical pole. The cytoplasm residues were left in the transient gap as indicated by positive signals for activated caspase-3 (Figure 1c, C and D). The similar comet-like movement of the nucleus in the enterocyte shedding from the villus shaft in vivo was shown in Figure 1d. Taken together, our data suggested that the apoptotic extrusion with gap formation in vivo can be recapitulated ex vivo by tissue culturing, and the gap may be transiently filled with the cytoplasmic residues of the apoptotic cell itself. 
a

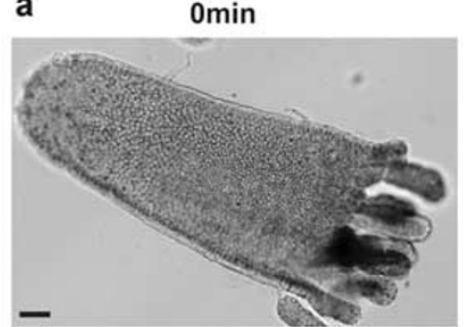

b

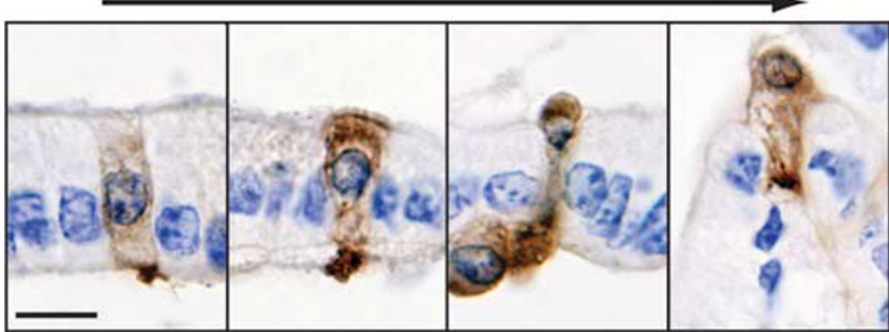

C

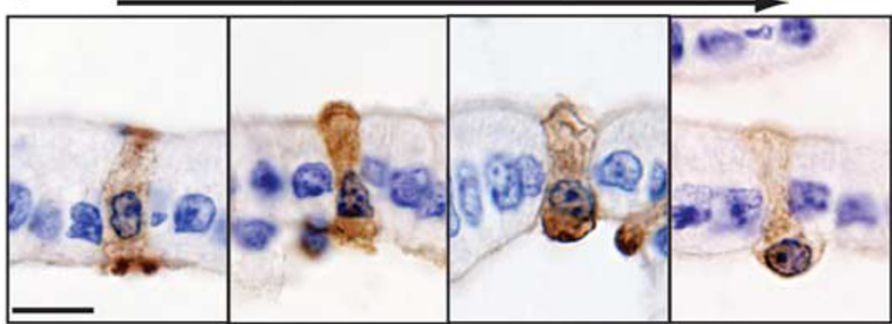

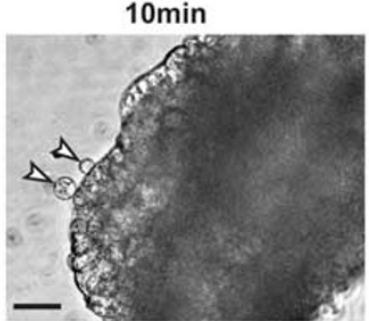

d
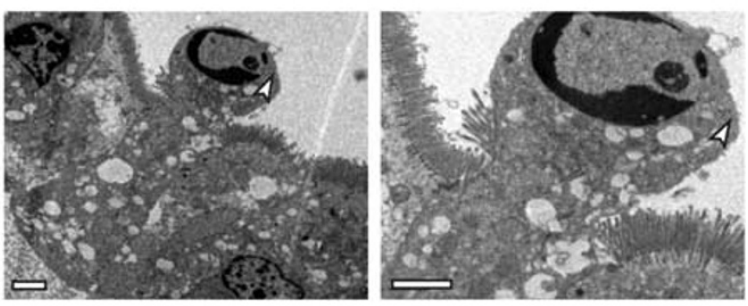

e
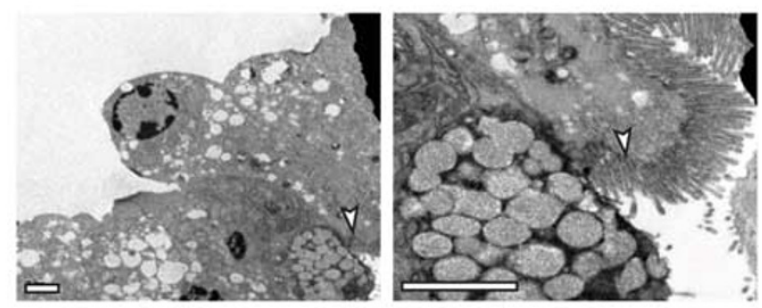

Figure 2 Cell shedding processes in the basal lamina-free villus unit ex vivo. (a) Freshly isolated villus-crypt units without basal lamina (left); villus-crypt units after incubation for $10 \mathrm{~min}$, arrows point to the apical extruding cell (right). Scale bars $=50 \mu \mathrm{m}$. (b, c) Cleaved caspase-3-positive cells in the different phases of extrusion toward the apical pole (b) and the basal pole (c) in the villus units after incubation for 7 min. Scale bars $=10 \mu \mathrm{m}$. (d, e) Cell shedding processes confirmed by transmission electron microscopy. (d) Typical cell shedding toward the apical pole. (e) A cell in the later stages of shedding with the nucleus beyond the basal pole of the neighboring cell. The apical surface and intercellular connection are magnified separately. Arrows mark the microvilli. Scale bars $=2 \mu \mathrm{m}$.

\section{Apoptotic Extrusion with a Gap Is Restricted to the Basal Lamina}

To explore the role of the lamina propria in epithelial shedding, we used the isolated crypt-villus units without lamina propria, a basal lamina-free model. The apical extrusion of cells was observed after culturing for 7-15 min (Figure 2a), whereas the frequency was decreased compared with a model where the basal lamina was present (data not shown). Interestingly, the nucleus of apoptotic cells may move toward either the apical (Figure 2b) or basal pole (Figure 2c) in the absence of the basal lamina. In addition, the shedding polarity was also observed with an electron microscope. The microvilli, mainly consisting of actin filaments, were broken down rapidly in an apical shedding manner (Figure 2d). In contrast, they can be partially retained during basal shedding at a given shedding phase (Figure 2e), indicating a different direction of cytoskeletal reorganization. The frequency of shedding toward the apical pole was relatively low (79/420, $19 \%$ ), which may be an underestimation because apoptotic cells during apical shedding were easily lost compared with basal shedding during tissue processing. In contrast, basal extrusion was not identified in the sections of villus units with basal lamina we scanned ( $n>200$ sections). Taken together, our data suggest that the cell shedding is bidirectional, but apical shedding is preferred in the presence of the basal membrane, possibly providing a physical barrier against basal extrusion and signaling the apoptotic cells to shed apically.

\section{Actin Reorganization During Apoptotic Cell Shedding}

We further investigated how the extruding force was generated. As the cells may reorganize their cytoskeletons quickly in response to mechanical forces, ${ }^{18,19}$ we added a resistant force against extrusion by embedding the villi into matrigel to augment the degree of potential cytoskeleton reorganization. Interestingly, in the en face view of the cultured villi, the actin accumulated at the interface between the apoptotic and live neighboring enterocytes was identified by phalloidin staining (Figure 3a). However, the highest level of phalloidin staining usually corresponded to increased expression of 
a
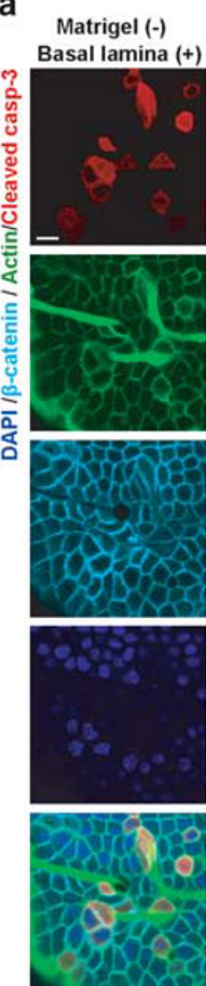

Matrigel (+)
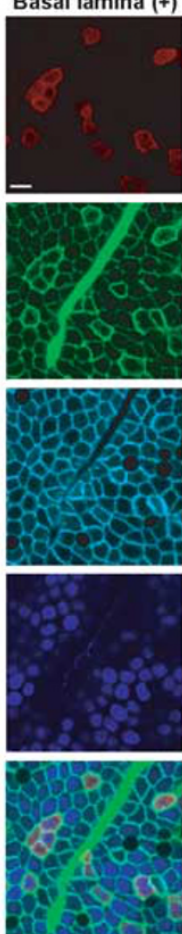

Matrigel (-)
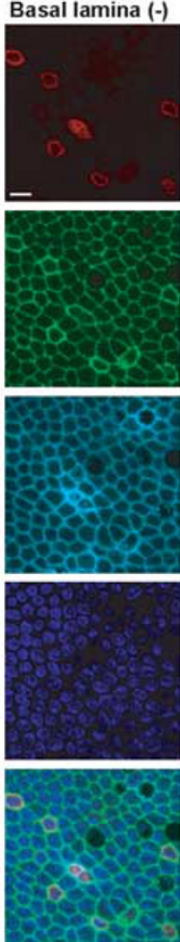

Matrigel $(+)$

Basal lamina (-)
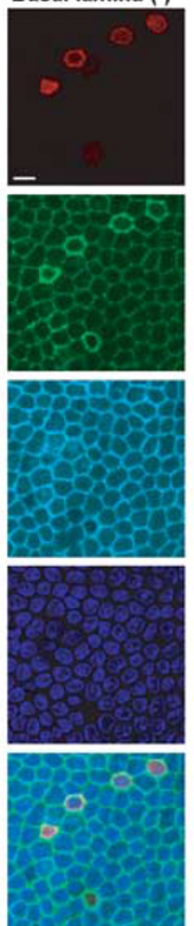

b
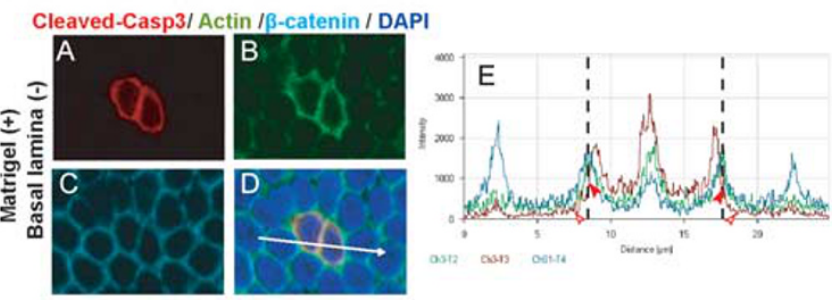

C Cleaved-Casp3/ Actin / DAPI
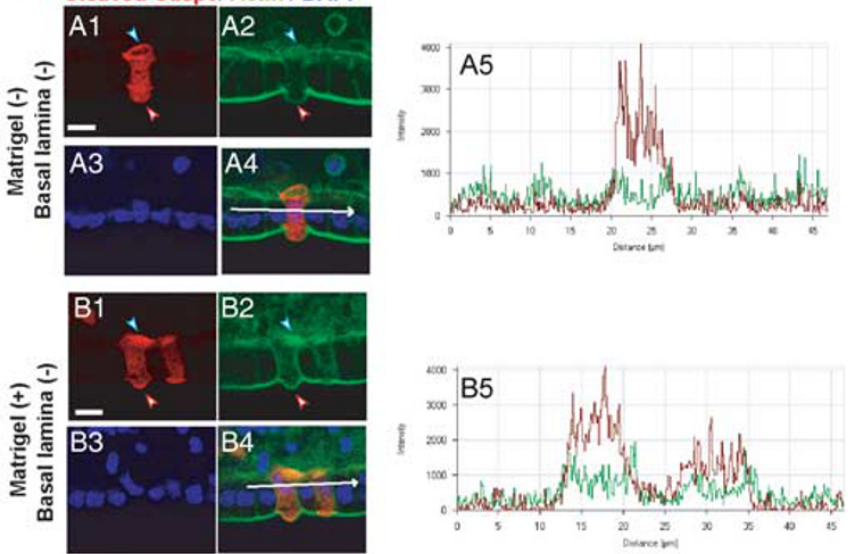

d

Cleaved-Casp3/ Actin / $\beta$-catenin / DAPI Matrigel (+) Basal lamina (-)
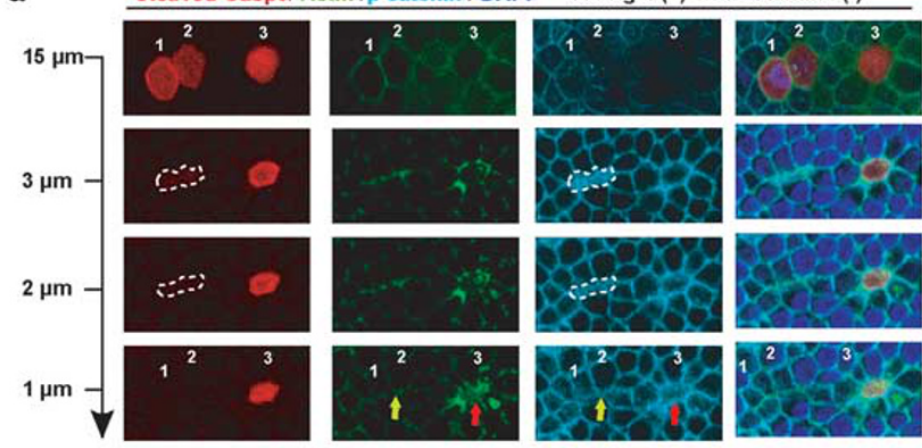

e

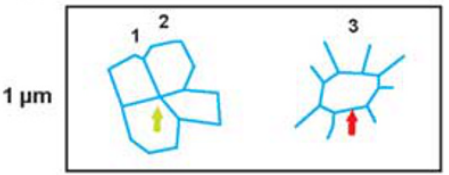

Figure 3 Actin reorganization during apoptotic cell shedding. (a) The actin ring at the interface of apoptotic and live neighboring enterocytes in the villus units with or without basal lamina cultured in the media, or cultured in matrigel. Observation is in the en face view. (b) Representative actin accumulation presented at the interface between two apoptotic enterocytes in the basal lamina-free villus units cultured in matrigel. Fluorescence intensity of F-actin (green), cleaved-caspase-3 (red) and $\beta$-catenin (blue) across two contacting apoptotic enterocytes and their live neighboring cells are marked by the white line. (E) The peak value of fluorescence intensity of F-actin (vertical dashed lines) overlapped with the increased cleaved-caspase-3 (arrowheads). Note the low expression of cleaved-caspase-3 in the live neighboring cells (open arrowheads). (c) Representative vertical sections of the extruding cell in basal laminafree villus units cultured in media (A) or matrigel (B). The apoptotic cell's budding was partially blocked in the villus unit cultured in matrigel (arrows). (d) Apoptotic cells in basal-lamina-free villus units cultured in matrigel. The ruler shows the downward distances of each optical section from the apical surface of the extruding cells. The dashed line indicates the boundary of two contacting apoptotic cells (1 and 2). The fluorescence of actin accumulation disappeared in the plane through which only the extended neighboring cells were scanned when the gap was closed (green arrow), whereas actin accumulation was observed at the basal pole of apoptotic cells that were not occupied by the cytoplasm extension of neighboring cells (red arrow). (e) Schematic representation showing the extension of live neighboring cells beneath apoptotic cells according to the $1 \mu \mathrm{m}$ scanning plane in (d). Scale bars $=10 \mu \mathrm{m}$.

activated caspase-3 $(97 \%, 35 / 36, n=3$; Figure $3 \mathrm{~b}, \mathrm{E})$, indicating that the actin accumulation mainly occurs in the cortical compartment of the apoptotic cell rather than in the live neighboring cells. Additionally, actin accumulation may occur at the interface of two contacting apoptotic cells (Figure 3b), indicating that the cytoskeleton reorganization of the apoptotic cells is independent of the live status of the neighboring cells. The observation in the vertical plane also 

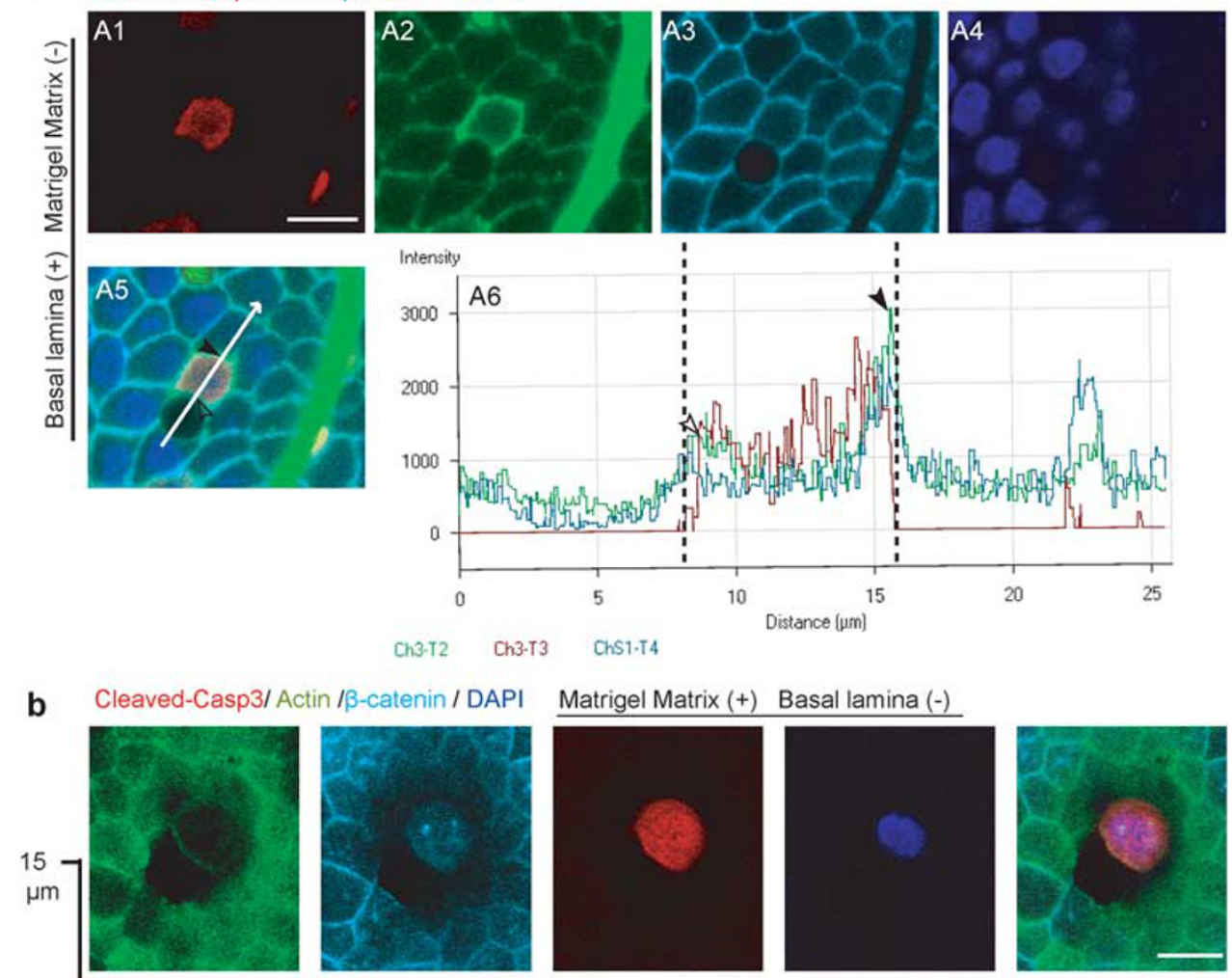

Ch3-T2 Ch3.T3 ChS1-T4
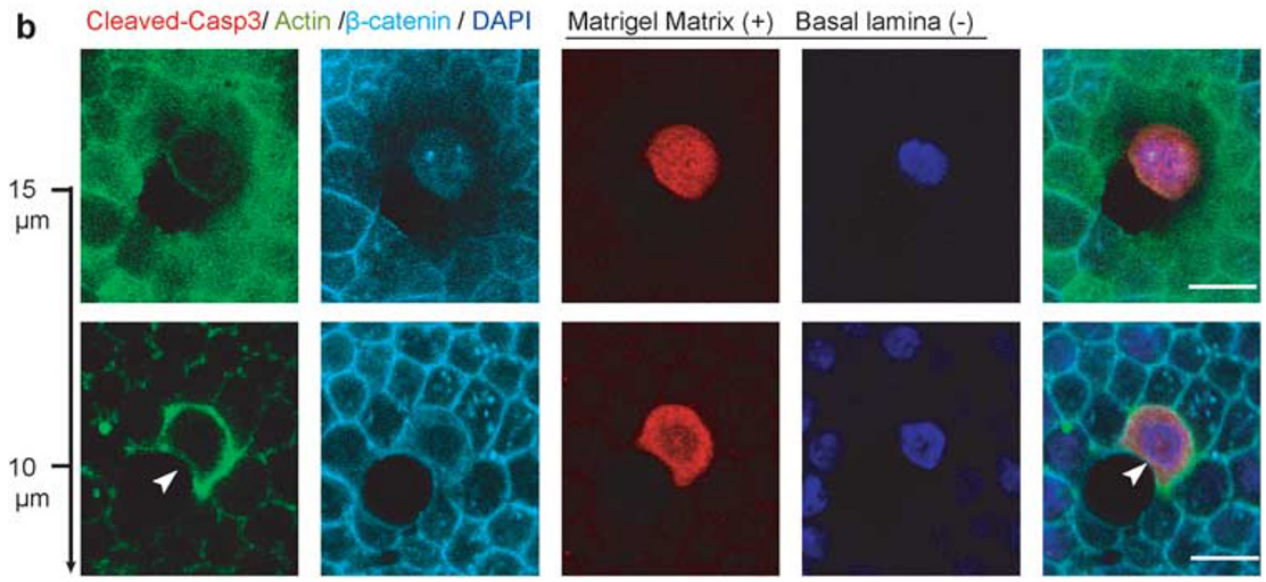

Figure 4 Shedding of apoptotic enterocytes neighboring goblet cells. (a) Representative apoptotic enterocytes neighboring goblet cells in the early shedding phase in the villus units with basal lamina cultured in the media. Fluorescence intensity of F-actin (green), cleaved-caspase-3 (red) and $\beta$-catenin (blue) in a goblet cell, an apoptotic enterocyte and a normal enterocyte are marked by the white line (A5). Vertical dashed lines in (A6) marked the boundary of apoptotic cells, with a higher level of cleaved caspase-3 compared with normal neighboring cells. The F-actin staining level in the interface between the goblet cell and the apoptotic enterocyte (open arrowhead) is much lower than that between the apoptotic enterocyte and the normal enterocyte (closed arrowhead). (b) Representative apoptotic enterocytes neighboring goblet cells in the later shedding phase in basal lamina-free villus units cultured in matrigel. The ruler shows the downward distances of each optical section from the basal surface of the extruding cells. The arrow indicates the cortical actin of the apoptotic cell. Note that most of the nucleus of apoptotic cells budded off the epithelia.

showed that actin accumulation was increased preferentially inside the apoptotic cells but not in the surrounding neighbors in the presence of resistance forces (Figure 3c). Additionally, we could not observe actin accumulation in the extended cytoplasm of neighboring cells beneath the extruding cell (Figure $3 \mathrm{~d}$ and e). Taken together, these data suggest that the increased F-actin structures (Figure 3a) we observed were mainly composed of the cortical compartment of apoptotic cells.

\section{Neighboring Goblet Cells Cannot Inhibit the Shedding of the Apoptotic Enterocyte}

Another major cell type in the villus epithelium is the goblet cell, characterized by mucin granules occupying the majority of the intracellular space. The cup-like shape in the cytoplasm, known as the theta, consists of three layers of cytoskeleton, a layer of microtubules and two layers of intermediate filaments. However, actin filaments are not prominent constituents in this area and contribute little in maintaining the shape of theta. ${ }^{20,21}$ We also found that the fluorescence staining for F-actin, in the interface between the goblet cell and normal enterocyte, was significantly lower than that between normal enterocytes (Figure 4a). Staining for $\beta$-catenin and E-cadherin, which may form a complex with actin, was also decreased at the interface of the goblet/ enterocyte (data not shown). Consistently, it is clear that contacting with the apoptotic enterocyte cannot lead to actin accumulation inside the goblet cell, and F-actin staining at 
the interface between the goblet cell and the apoptotic enterocyte is lower than that between the normal enterocyte and the apoptotic enterocyte (Figure 4a). The neighboring goblet cell cannot inhibit the apoptotic shedding of enterocytes, even after adding resistance force by culturing in matrigel (Figure 4b). Taken together, these data provide further evidence that the actin reorganization mainly occurred in the apoptotic cell, and the cytoskeleton reorganization in apoptotic cells rather than in live neighboring cells may provide the major force for apoptotic extrusion.

\section{Apoptotic Cell Deformation and Gap Closure}

Cell shrinkage or AVD was found to precede cytochrome $c$ release, caspase-3 activation and other apoptotic events, an early prerequisite of apoptosis. ${ }^{4}$ Consistently, it may help compact the apoptotic cell as the neighboring cells squeeze the cells out of the space. ${ }^{7}$ However, in our system, using three-dimensional projection, we failed to find the obvious AVD of the apoptotic enterocytes during the early shedding phase (Figure 5a). In contrast, the height of the apoptotic cell was significantly increased compared with the surrounding cells during the phase of cell shedding without the extension of neighboring cells, and the apoptotic cell volume appeared to increase (Figure 5a). Whether shedding apically or basally in the basal lamina-free model, the apoptotic cell may distend toward both the apical and basal poles (Figure $5 \mathrm{~b}$ ). The movement of the nucleus inside the cytoplasm was found in all the elongated apoptotic cells; moreover, the stretching and deformation of the nucleus was occasionally observed (Supplementary Figure S2). All these data suggest that the forces along the apicobasal axis were generated inside apoptotic cells to stretch out the cell itself (Figure 5d).

Previous studies suggest that the actomyosin ring formed in live neighboring cells may contract together from one pole, which ensures the parallels between extrusion and gap closure. ${ }^{22,23}$ We found that the apoptotic cells shrunk and the interior interface of the gap was covered by the apical surface of the live neighboring cells in the later phase of cell shedding (Figure $5 \mathrm{c}$ ), suggesting a process of gradually separating the apoptotic cell from the basal membrane and its neighbors.
This separation may be caused by the numerous vacuoles that form in the apoptotic cell. ${ }^{24}$ Regardless of whether the apoptotic cells shed apically or basally in the basal laminafree model, gap closure was initiated around the middle of the cell (Figure 5b, A), indicating the absence of a contraction ring at either the apical or basal pole of the living neighboring cells. All these data suggest that gap closure does not depend on the formation and contraction of an actomyosin ring in live neighboring cells.

\section{DISCUSSION}

The mucosal epithelium is the center of interactions between the mucosal immune system and luminal contents. ${ }^{25}$ The intestinal barrier is sustained during the high rate of normal cell turnover in the epithelium through unknown mechanisms. Previous studies based on in vitro studies suggest that the cell destined for apoptosis signals its live neighboring epithelial cells to form an actomyosin ring, whose contraction not only squeezes the dying cell out of the epithelial sheet but also closes any gaps that may have resulted from the dying cell's exit. ${ }^{2,7}$ However, studies based on in vivo observations from a multiphoton microscope provided direct evidence that intestinal epithelial shedding may leave a transient gap. ${ }^{13,14}$ In addition, shedding cells leave the epithelium tangential to the plane of the epithelium at a speed of $0.83 \pm 0.6 \mu \mathrm{m} / \mathrm{min},{ }^{13}$ which is significantly faster than apoptotic cell extrusion in vitro, which takes over an hour. ${ }^{7}$ The flatter morphology instead of the column shape, and adhesion to rigid substrates instead of flexible substrates by the cell lines may have an impact on the apoptotic shedding process. ${ }^{2}$

In our present model, the nucleus of apoptotic cells budded out of the monolayer with a time course similar to in vivo epithelial shedding. Gap formation was also observed under a confocal microscope. Using the fluorescent caspase substrate, PhiPhiLux, Watson et $a l^{13}$ found that full caspase-3 activation is not an uncommon event before cell shedding. Their work was based on human surgical histological specimens demonstrating that most shedding cells were for activated caspase-3, indicating the different sensitivities of these

Figure 5 Apoptotic cell deformation and gap closure. (a) The extruding cells in basal lamina-containing villus units were cultured for 7 min in media. (A, B) Three-dimensional confocal reconstructions; the arrows point to the area without microvilli in the environment. (C1-2) Two representative sections of the three-dimensional reconstructions with $5 \mu \mathrm{m}$ interval. The height of the apoptotic cells is significantly higher than that of the live neighboring cells $(n=6, P<0.01$, $t$-test). (b, A1-2) Representative three-dimensional confocal reconstructions of the shedding cells with different polarity. Five apoptotic cells are numerically labeled. Arrowheads in (A) show gap closure in the middle of the interface of the third and fifth apoptotic cell. (B) A representative section of the three-dimensional reconstruction. The basal-lateral surface of the cells neighboring the third, fourth and fifth apoptotic cells became a semi-spherical surface. (c) Representative cell shedding in the late shedding phase in the villus units with basal lamina. (A) The consecutive vertical sections with the ruler showing the downward distances of each optical section from the basal surface of the shedding cell. Only the tiny areas contacting with live neighboring cells (arrowheads in the $10 \mu \mathrm{m}$ and $11 \mu \mathrm{m}$ planes in (A) remained. (B) The three-dimensional confocal reconstructions of the shedding cell. The dashed line in (B2) indicates the outline of the apoptotic cell. The internal area of the gap was mainly covered by the apical surface of the microvilli from live neighboring cells, which can be seen from the fluorescence of the phalloidin (dashed curved lines in the $8 \mu \mathrm{m}$ plane in A and arrows in B2). The nuclei of neighboring cells were not in the same plane. (d) The left graph shows the models of apoptotic cell deformation in the villus units with or without basal membrane. The right graph shows the models of gap closure. Arrows indicate the proposed direction of applied force. The numbers labeling the dashed lines in (a-c) indicate the cell length quantified by Zeiss LSM software. Asterisks in $(\mathbf{a}, \mathbf{b})$ indicate the area with lower fluorescence because of vesicles generated in the cytoplasm of apoptotic cells. Scale bars $=10 \mu \mathrm{m}$. 
two methods. ${ }^{26}$ In addition, they found that some morphologically normal cells were positive for activated caspase- $3 .{ }^{26}$ Similarly, we also found weak caspase- 3 expression confined to the cytoplasm in the very early phases of cell shedding (Figure 1). It remains unclear whether cell shedding occurs before or after caspase cleavage. Altogether, these data strongly suggest that epithelial shedding in our ex vivo model shares similar mechanisms with that in vivo. Cultured villus units with adequate shedding cells can be fixed and tested directly, leading to better preservation of shedding cells and a greater ability to study cytoskeletal reorganization compared with a reported method. ${ }^{26}$

The effete or abnormal intestinal epithelial cells are not just displaced out of the epithelial layer by the migration of a

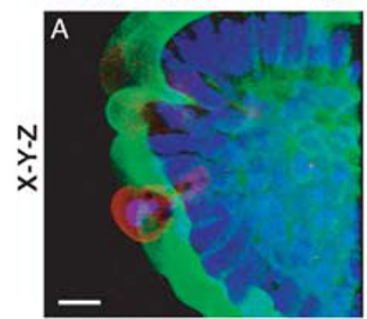

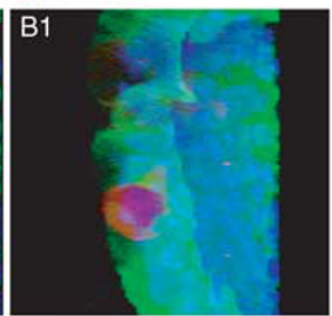

b Cleaved-Casp3/Actin / DAPI
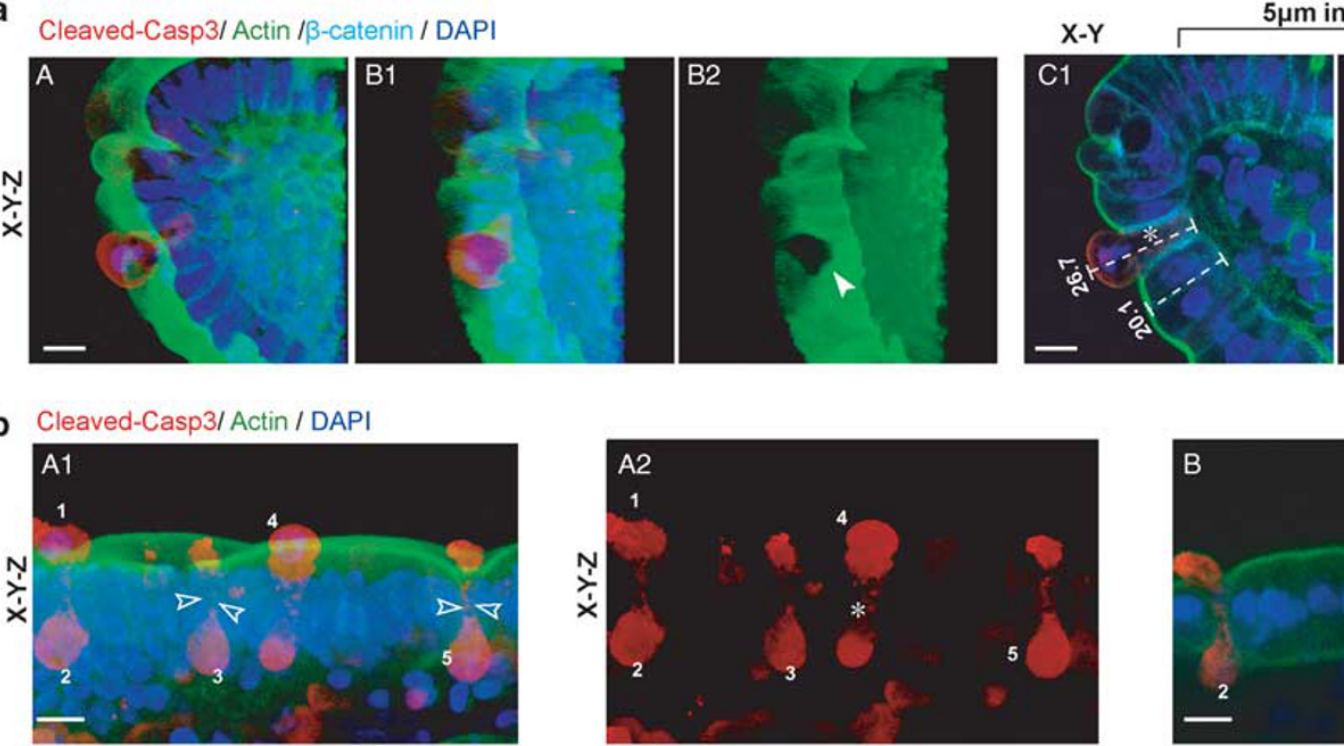

$5 \mu \mathrm{m}$ interval

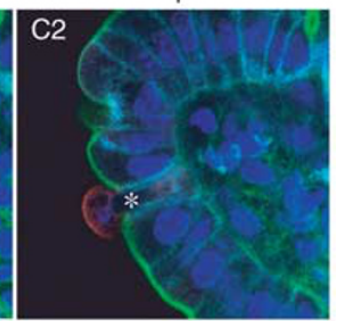

$X-Y$
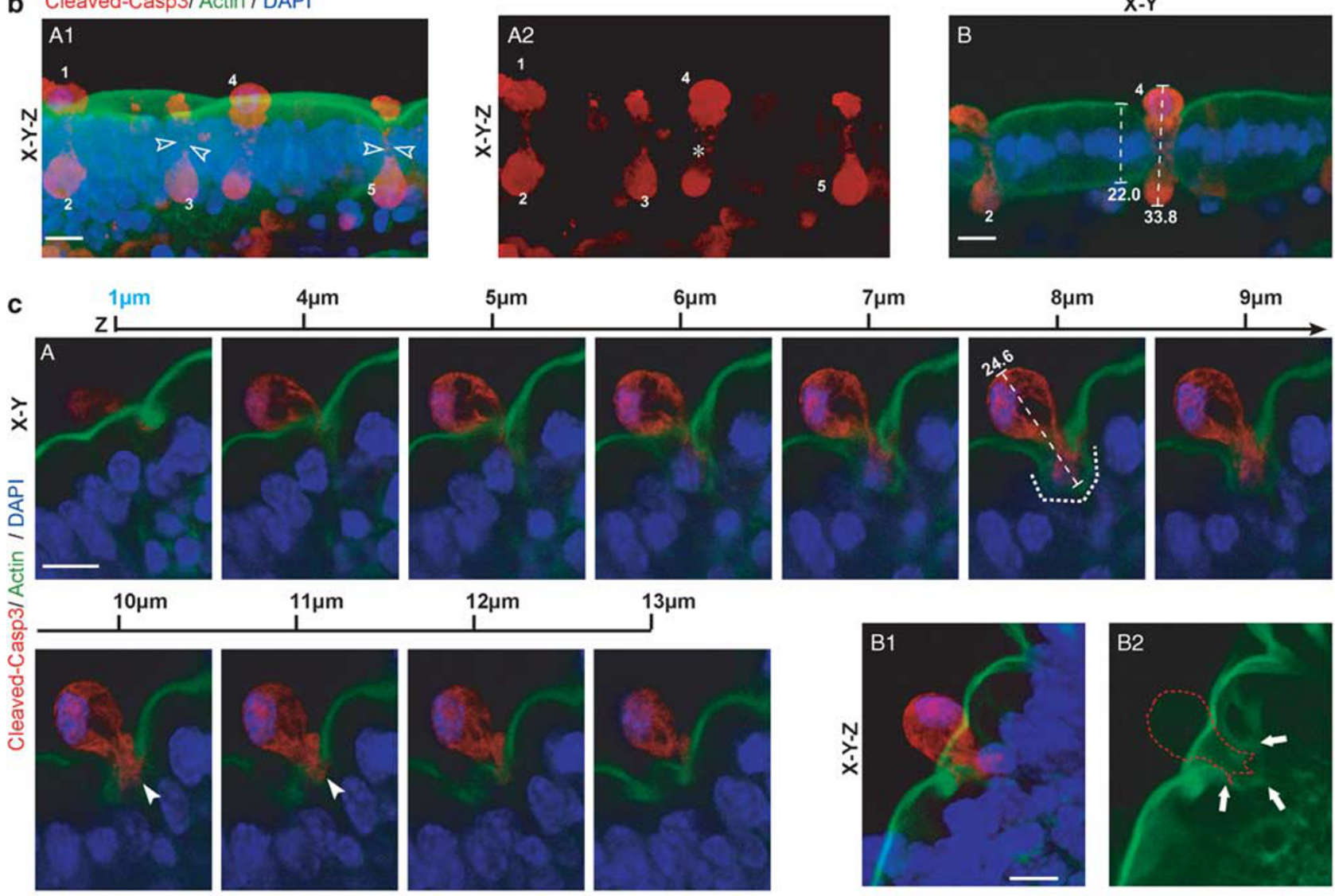

d

Apoptotic Cell Deformation
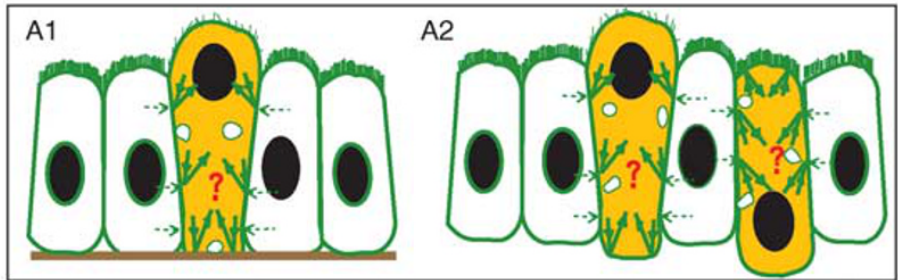

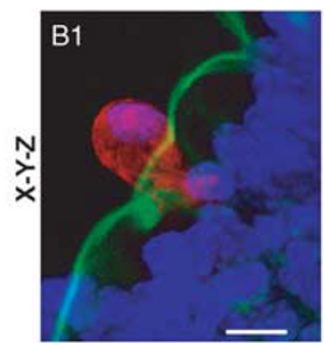

B2

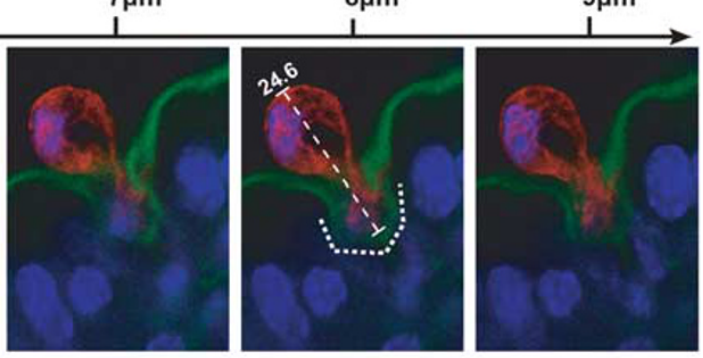

Gap Closure

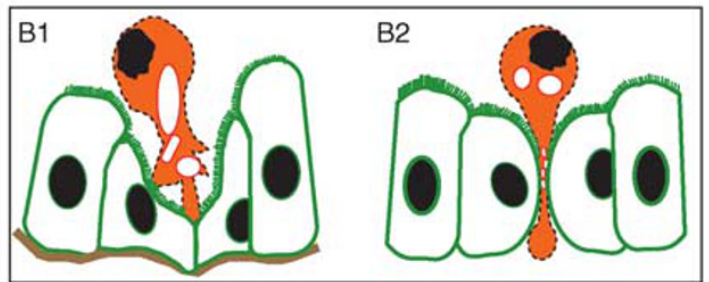


cells lower down the villus. ${ }^{27}$ An unknown force was thought to push the shedding cells out. ${ }^{27}$ The force was thought to be generated by the contraction of the actomyosin ring formed in the cells surrounding apoptotic cells. ${ }^{2,7}$ However, we found obvious actin reorganization in the apoptotic enterocyte but not in the live neighboring cells. Our findings are consistent with the restitution of single-cell defects in the mouse colon, in which no actin ring surrounding the lesion was found. ${ }^{17}$ The considerably high speed of cell shedding and threedimensional culture made it impossible to microinject inhibitors into the live neighboring cells in order to block actin reorganization. The apoptotic cell can complete the shedding process when neighboring a goblet cell, in which the majority were occupied by mucin granules but lacked F-actin. ${ }^{20,21}$ All these data suggest that the major pushing force does not come from the squeezing of live neighboring cells. Secretion or lymphatic obstruction has been reported to increase epithelial cell shedding, suggesting that fluid flow or hydrostatic pressure originating within the core of the villus may create a driving force to push epithelial cells out of the villus monolayer. ${ }^{27,28}$ In the basal lamina-free model, our findings clearly showed that the epithelial shedding does not need the force generated in the core of the villus.

The active cell extrusion was observed in the MDCK epithelial cells that express constitutively active oncogenic Ras (RasV12) or v-Src, and are surrounded by normal cells. They may first increase their cell height, without the activation of apoptotic pathways. ${ }^{29,30}$ We also found that the enterocytes with caspase- 3 activation may increase their height (Figure 5a and $b$ ). The nuclear movement in the early shedding phase does not require the extension of neighboring cells and dissociation with live neighboring cells and basal lamina. Additionally, the nucleus can also become deformed and elongate (Supplementary Figure S2), similar to the deformations seen in cancer cells. ${ }^{31}$ Together with the above mentioned F-actin reorganization in the apoptotic cell, our data strongly suggest that apoptotic cells may firstly undergo an active deformation process (Figure $5 \mathrm{~d}$ ). In the passive extrusion model, localization of the actomyosin contractile ring dictates the extrusion direction. ${ }^{2}$ Obviously, this theory cannot explain the loss of polarity during cell deformation in our model.

Cell shape was controlled by a set of intracellular and extracellular forces. ${ }^{32}$ The active deformation of apoptotic cells was followed by cell shrinkage and dissociation of the basal lamina and neighboring cells, leading to an imbalance between extracellular forces and the intracellular forces in the live neighboring cells. In the basal lamina-free model, we found that in the later shedding phases the basal-lateral surface of the neighboring cells became semispherical and the gap was narrowed (Figure 5b). This is consistent with the spherical morphology of the isolated cells, which were shaped by cortical tension to reduce the surface area. ${ }^{32}$ The control of cell shape also involves an organization of the total plasma membrane into an effective surface of extracellular matrix. ${ }^{32}$
In the incubated villus units with basal lamina, we observed depression in the apical surface of live neighboring cells and bending of the basal lamina during gap closure. The depression on the villi surface was more obvious after the shedding of masses of apoptotic cells (Supplementary Figure S1), which is consistent with the clefts formed at the villus tip. ${ }^{24}$ Therefore, our data support a potential model for apoptotic epithelial cell clearance. Apoptotic cells firstly deform and are gradually separated from the basal membrane and surrounding cells. Live neighboring cells, sensing the change in extracellular forces, extend to restitute the vacant area, a process accompanied by the bending of a local basal membrane (Figure 5d).

It is noteworthy that the nuclei of apoptotic cells budded off the epithelia may be shed easily during tissue processing, leaving cytoplasmic residues in the transient gap (Supplementary Figure S3). Therefore, we speculate that this kind of shedding is easier in vivo. Previously, studies have shown that beneath the shedding cell there is an area of reduced eosin staining that does not contain a nucleus, corresponding to the epithelial gaps observed by confocal microscopy in the living mouse intestine. ${ }^{26}$ Consistently, we identified that the cytoplasmic residue that filled the gap contains many vesicles (Figure $5 \mathrm{a}$ and $\mathrm{b}$ ). As previously reported, ${ }^{26}$ it was thought that the cytoplasmic residue may be the substance plugging epithelial gaps following cell shedding in vivo.

In conclusion, we propose an ex vivo model for investigating intestinal epithelial shedding with gap formation. Our findings provide new insights into the mechanisms leading to cell shedding, and highlight the roles of active deformation of apoptotic cells in clearing itself. In addition, adhesion-restricted shedding polarity may serve as a protective mechanism against the metastasis of unhealthy cells into tissue. Our results yield new information on the pathogenesis of intestinal disorders that result from altered epithelial shedding control and barrier function.

Supplementary Information accompanies the paper on the Laboratory Investigation website (http://www.laboratoryinvestigation.org)

\section{ACKNOWLEDGEMENTS}

We gratefully acknowledge Dr Tianmin Cheng, Dr Jia Cao, Dr Chunmeng Shi and Dr Kexi Yi for advice; Mingsong Feng, Yunmu Li, Yu Ning, Mingqiang Shen and Qing Zhou for technical assistance; and Yang Xiang for technical help with LSM confocal microscope. This work was supported by the National Basic Science and Development Program (973 Program, no. 2005CB522605); the Innovation Group of Education Ministry (Grant no. IRT0712); and the Opening Foundation of National Laboratory of Trauma, Burns and Combined Injury (SKLZZ200911).

\section{DISCLOSURE/CONFLICT OF INTEREST}

The authors declare no conflict of interest.

1. Bement WM. Actomyosin rings: the riddle of the sphincter. Curr Biol 2002;12:R12-R14.

2. Slattum G, McGee KM, Rosenblatt J. P115 RhoGEF and microtubules decide the direction apoptotic cells extrude from an epithelium. J Cell Biol 2009;186:693-702. 
3. Hengartner MO. The biochemistry of apoptosis. Nature 2000;407: 770-776.

4. Maeno $\mathrm{E}$, Ishizaki $\mathrm{Y}$, Kanaseki $\mathrm{T}$, et al. Normotonic cell shrinkage because of disordered volume regulation is an early prerequisite to apoptosis. Proc Natl Acad Sci USA 2000;97:9487-9492.

5. Gomez-Angelats M, Cidlowski JA. Cell volume control and signal transduction in apoptosis. Toxicol Pathol 2002;30:541-551.

6. Bortner CD, Cidlowski JA. Uncoupling cell shrinkage from apoptosis reveals that $\mathrm{Na}+$ influx is required for volume loss during programmed cell death. J Biol Chem 2003;278:39176-39184.

7. Rosenblatt J, Raff MC, Cramer LP. An epithelial cell destined for apoptosis signals its neighbors to extrude it by an actin- and myosindependent mechanism. Curr Biol 2001;11:1847-1857.

8. Vidal $M$, Larson DE, Cagan RL. Csk-deficient boundary cells are eliminated from normal Drosophila epithelia by exclusion, migration, and apoptosis. Dev Cell 2006;10:33-44.

9. Ninov N, Chiarelli DA, Martin-Blanco E. Extrinsic and intrinsic mechanisms directing epithelial cell sheet replacement during Drosophila metamorphosis. Development 2007;134:367-379.

10. Mayhew TM, Myklebust R, Whybrow A, et al. Epithelial integrity, cell death and cell loss in mammalian small intestine. Histol Histopathol 1999;14:257-267.

11. Lotz MM, Rabinovitz I, Mercurio AM. Intestinal restitution: progression of actin cytoskeleton rearrangements and integrin function in a model of epithelial wound healing. Am J Pathol 2000;156: 985-996.

12. Marchiando AM, Graham WV, Turner JR. Epithelial barriers in homeostasis and disease. Annu Rev Pathol 2010;5:119-144.

13. Watson AJ, Chu S, Sieck $L$, et al. Epithelial barrier function in vivo is sustained despite gaps in epithelial layers. Gastroenterology 2005:129:902-912.

14. Kiesslich R, Goetz M, Angus EM, et al. Identification of epithelial gaps in human small and large intestine by confocal endomicroscopy. Gastroenterology 2007;133:1769-1778.

15. Wang F, Wang J, Liu D, et al. Normalizing genes for real-time polymerase chain reaction in epithelial and nonepithelial cells of mouse small intestine. Anal Biochem 2010;399:211-217.

16. Liu D, Wang F, Zou Z, et al. Long-term repopulation effects of donor BMDCs on intestinal epithelium. Dig Dis Sci 2010;55: 2182-2193.
17. Gunzel D, Florian P, Richter JF, et al. Restitution of single-cell defects in the mouse colon epithelium differs from that of cultured cells. Am J Physiol Regul Integr Comp Physiol 2006;290:R1496-R1507.

18. Pollard TD, Cooper JA. Actin, a central player in cell shape and movement. Science 2009;326:1208-1212.

19. Fletcher DA, Mullins RD. Cell mechanics and the cytoskeleton. Nature 2010;463:485-492

20. Specian RD, Neutra MR. Cytoskeleton of intestinal goblet cells in rabbit and monkey. The theca. Gastroenterology 1984;87:1313-1325.

21. Oliver MG, Specian RD. Cytoskeleton of intestinal goblet cells: role of actin filaments in baseline secretion. Am J Physiol 1990;259(6 Pt 1):G991-G997.

22. Florian P, Schoneberg T, Schulzke JD, et al. Single-cell epithelial defects close rapidly by an actinomyosin purse string mechanism with functional tight junctions. J Physiol 2002;545(Pt 2):485-499.

23. Bement WM, Forscher P, Mooseker MS. A novel cytoskeletal structure involved in purse string wound closure and cell polarity maintenance. J Cell Biol 1993;121:565-578.

24. Potten CS, Allen TD. Ultrastructure of cell loss in intestinal mucosa. J Ultrastruct Res 1977;60:272-277.

25. Turner JR. Intestinal mucosal barrier function in health and disease. Nat Rev Immunol 2009;9:799-809.

26. Bullen TF, Forrest S, Campbell F, et al. Characterization of epithelial cell shedding from human small intestine. Lab Invest 2006;86:1052-1063.

27. Watson AJ, Duckworth CA, Guan Y, et al. Mechanisms of epithelial cell shedding in the Mammalian intestine and maintenance of barrier function. Ann NY Acad Sci 2009;1165:135-142.

28. Lee JS. Epithelial cell extrusion during fluid transport in canine small intestine. Am J Physiol 1977;232:E408-E414.

29. Kajita M, Hogan C, Harris AR, et al. Interaction with surrounding normal epithelial cells influences signalling pathways and behaviour of Srctransformed cells. J Cell Sci 2010;123(Pt 2):171-180.

30. Hogan C, Dupre-Crochet $S$, Norman $M$, et al. Characterization of the interface between normal and transformed epithelial cells. Nat Cell Biol 2009;11:460-467.

31. Yamauchi K, Yang $M$, Jiang $P$, et al. Real-time in vivo dual-color imaging of intracapillary cancer cell and nucleus deformation and migration. Cancer Res 2005;65:4246-4252.

32. Lecuit T, Lenne PF. Cell surface mechanics and the control of cell shape, tissue patterns and morphogenesis. Nat Rev Mol Cell Biol 2007;8:633-644. 